\title{
Results of repeat manipulation under ultrasound-guided cervical nerve root block with corticosteroid and local anaesthetic injection for recurrence of frozen shoulder
}

\author{
Tatsuki Oshiro ${ }^{1 *}$, Masayoshi Yagi ${ }^{1}$, Kazuki Harada ${ }^{1}$ and Kieun Park ${ }^{2}$
}

\begin{abstract}
Background: This study aimed to evaluate the clinical results of a repeat manipulation under ultrasound-guided cervical nerve root block (MUC) with corticosteroid and local anaesthetic injection for recurrence of idiopathic frozen shoulder after MUC.

Methods: A consecutive series of 42 shoulders in 39 patients with idiopathic frozen shoulder underwent MUC. All patients were assessed according to the American Shoulder Elbow Surgeon (ASES) score and shoulder range of motion (ROM) both before MUC and at 1 year thereafter. If patients continued to have pain and limited ROM at 3 months after the procedure, they were offered a repeat MUC. Such patients were also assessed before the procedure and at 3 months and 1 year thereafter.

Results: The initial MUC was successful in 31 shoulders (single group). Repeat MUC was required in 11 shoulders (repeat group). Patients in the single group showed significant improvement in ROM and ASES score at 1 year after the procedure $(p<0.001)$; similarly, patients in the repeat group had significant improvement in ROM and ASES score at 3 months and 1 year after the procedure $(p<0.001)$. Patients in the repeat group had had significantly more severely limited ROM $(p<0.01)$ and decreased ASES score $(p<0.001)$ before the procedure compared with those in the single group.

Conclusions: A repeat MUC with corticosteroid and local anaesthetic injection is a valuable option before proceeding to surgery for recurrence of idiopathic frozen shoulder. When there is severely limited ROM and decreased ASES score before the MUC, a repeat MUC may be necessary, which would require the patient's informed consent.
\end{abstract}

Trial registration: Retrospectively registered

Keywords: Idiopathic frozen shoulder, MUC, Repeat MUC

\footnotetext{
* Correspondence: tatsuki.pt34@gmail.com

'Yagi Orthopaedic Clinic, 47-1, Zike-machi, Kakogawa-cho, Kakogawa, Hyogo 675-0066, Japan

Full list of author information is available at the end of the article
}

(c) The Author(s). 2020 Open Access This article is licensed under a Creative Commons Attribution 4.0 International License, which permits use, sharing, adaptation, distribution and reproduction in any medium or format, as long as you give appropriate credit to the original author(s) and the source, provide a link to the Creative Commons licence, and indicate if changes were made. The images or other third party material in this article are included in the article's Creative Commons licence, unless indicated otherwise in a credit line to the material. If material is not included in the article's Creative Commons licence and your intended use is not permitted by statutory regulation or exceeds the permitted use, you will need to obtain permission directly from the copyright holder. To view a copy of this licence, visit http://creativecommons.org/licenses/by/4.0/. The Creative Commons Public Domain Dedication waiver (http://creativecommons.org/publicdomain/zero/1.0/) applies to the data made available in this article, unless otherwise stated in a credit line to the data. 


\section{Background}

Idiopathic frozen shoulder is characterised by a limited range of motion (ROM) and shoulder pain [1]. Although the symptoms of the disease are selflimiting [2, 3], over $50 \%$ of patients treated nonoperatively for idiopathic frozen shoulder still have symptoms and impaired range of motion (ROM) as much as 7 years after initial follow-up [4]. In cases with residual restriction of motion after conservative treatment, surgical intervention including open surgical release, arthroscopic capsular release and manipulation under anaesthesia (MUA) with or without corticosteroid and local anaesthetic injection may be indicated [5-16].

Recently, however, it has been reported that manipulation under ultrasound-guided cervical nerve root block (MUC) has resulted in good clinical results [17, 18]. However, while a limited number of studies have discussed recurrence in patients who had unsuccessful outcomes with MUC, to the best of our knowledge, no other studies have evaluated the clinical outcomes of repeated MUC (repeat MUC).

The purpose of this study was to evaluate the shortterm clinical outcomes of a repeat MUC with corticosteroid and local anaesthetic injection performed when the initial results were less than satisfactory or when patients developed recurrent symptoms after their initial MUC. We also looked at whether there were any differences between the single and repeat MUC groups before the procedure.

\section{Methods}

This was a retrospective case-control study, with a single surgeon and a consecutive series of patients who underwent MUC for idiopathic frozen shoulder between April 2013 and August 2019. The study was approved by the institutional review boards of the authors' affiliated institutions.

\section{Inclusion and exclusion criteria}

The criteria for MUC were as follows: (1) patients with pain and limited active and passive ROM in all three planes $\left(\leq 120^{\circ}\right.$ of forward flexion $[\mathrm{FF}]$ and abduction, $\leq$ $30^{\circ}$ of external rotation at the side [ER]); (2) patients who did not respond to conservative therapy such as a combination of non-steroidal anti-inflammatory drugs (NSAIDs), intra-articular steroid injections and physiotherapy for at least 3 months.

Exclusion criteria were as follows: (1) rotator cuff tear, (2) osteoarthritis of the shoulder, (3) any fracture involving the shoulder girdle, (4) diabetics with frozen shoulder and (4) failure to attend 1-year follow-up.

\section{Patient selection}

Forty-two patients (45 shoulders) who underwent MUC for frozen shoulder were evaluated. Of these 42 patients (45 shoulders), 3 patients were excluded due to absence of follow-up for at least 1 year, leaving 39 patients (42 shoulders) who were finally included in the study.

\section{MUC procedure}

MUC is performed with the patient in a supine position. The cervical nerve roots $(\mathrm{C} 5 / \mathrm{C} 6)$ between the anterior and middle scalene muscle are identified using ultrasound. The patient is then injected with $10 \mathrm{ml}$ of anapeine, $10 \mathrm{ml}$ of normal saline and $10 \mathrm{ml}$ of $1 \%$ lidocaine. The scapula is stabilised with one hand, while it is recommended that the other hand should hold the proximal portion of the humerus, using a short lever arm to avoid serious complication. The shoulder is manipulated sequentially through a range of abduction, external rotation, forward flexion and cross-body adduction followed by internal rotation flexion. Finally, the arm is extended and rotated internally. After the MUC procedure, $2 \mathrm{~mL}$ of $1 \%$ lidocaine plus $40 \mathrm{mg}$ of triamcinolone is injected into the joint to prevent postoperative pain.

\section{Postoperative physiotherapy}

All patients attended physiotherapy three times a week for at least 3 months. Such therapy included relaxation of the muscles around the shoulder girdle, glenohumeral mobilisation and passive capsular and muscle stretching were applied to avoid pain. At home, patients were instructed to follow through with a home flexibility programme once every 4 to $5 \mathrm{~h}$ for the first week after the procedure. The programme included five repetitions of passive stretches for flexion utilising a cane.

\section{Inclusion criteria for repeat MUC}

Patients who had a good response after their initial MUC but presented at a later date with recurrent symptoms or those whose initial MUC was less than satisfactory were offered a repeat MUC. Inclusion criteria for the repeat MUC were as follows: (1) patients who did not respond to physiotherapy for at least 3 months after MUC, (2) patients who continued to have pain defined as $5 / 10$ or greater on the visual analogue scale (VAS) for pain and (3) patients who had limited ROM in all three planes ( $\leq 135^{\circ}$ of $\mathrm{FF}$ and abduction, $\leq 35^{\circ}$ of ER).

\section{Outcome assessment}

All patients were assessed before the procedure and at 1 year after MUC. Outcome measures included ROM of the shoulder (FF, abduction and ER) and the American Shoulder Elbow Surgeons (ASES) score.

The ASES score includes two parameters: pain and daily life activities. The maximum score for pain was 50 
points (range, 0 to 10 ; where $0=$ no pain and $10=$ severe pain). Also, a maximum of 50 points were assigned for the ability to carry out daily activities. Therefore, the total ASES score was 100 points.

Patients who had a repeat MUC were also assessed before the procedure, at 3 months (i.e., before the repeat MUC) and at one year after the procedure.

\section{Statistical analysis}

We used unpaired $t$ test to compare the averages of continuous variables (such as age, duration of symptoms, ROM, ASES score) and chi-square tests to compare the proportion of categorical factors, such as sex, between the groups. The level of significance was set at $p<0.05$. All analyses were performed with the use of SPSS software for Windows (version 20).

\section{Results}

Of the 39 patients (42 shoulders), 31 patients were women $(79 \%)$ and 8 patients were men $(21 \%)$, with a mean age of $53 \pm 7$ years and a mean follow-up period of 15 months (range 12 to 36 months). Prior to the procedure, the average duration of symptoms was 8.5 months (range 6 to 21 months). There were 3 patients
(11\%) who had bilateral frozen shoulder at different times. All 39 patients were managed with a supervised physiotherapy programme for at least 3 months after MUC. After completion of the programme, 31 shoulders (74\%) showed decreased pain and improved motion (single group). The remaining 11 shoulders (26\%) continued to have both loss of motion and pain and required a repeat MUC (repeat group).

\section{Single group}

In the single group, there was a statistically significant improvement in ROM of forward flexion, external rotation, ASES scores and ASES score for pain from before the procedure to follow-up 1 year after MUC $(p<0.001)$ (Figs.1, 2, 3 and 4).

\section{Repeat group}

In the repeat group, there was an initial improvement in ROM of forward flexion, external rotation, ASES score and ASES score for pain, which subsequently deteriorated by the time of the repeat MUC, after which ROM, ASES scores and ASES score for pain improved significantly $(p<0.001)$ (Figs.1, 2, 3 and 4$)$.

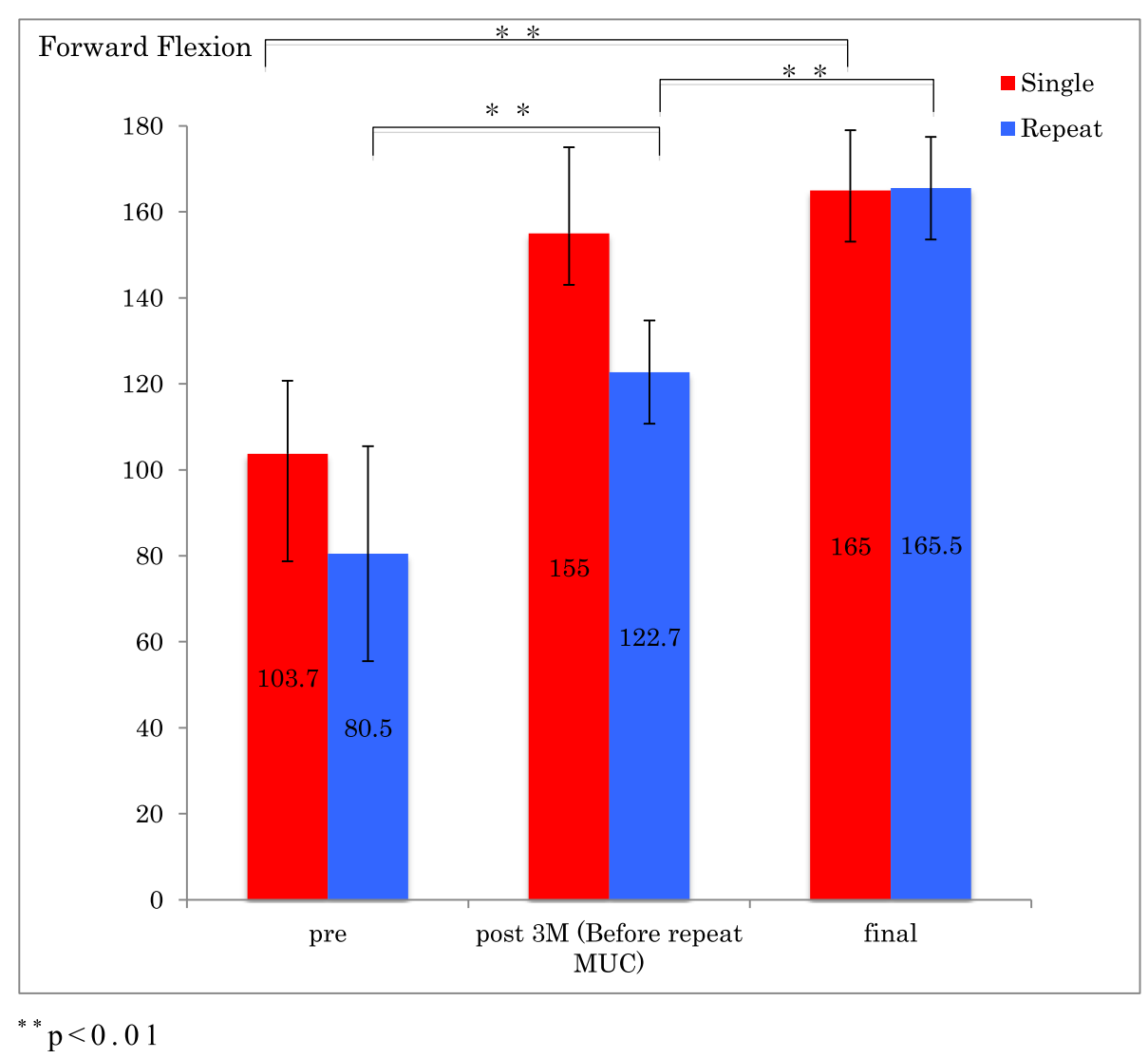

Fig 1 Forward flexion before and after single MUC and repeat MUC. ${ }^{* *} p<0.01$ 


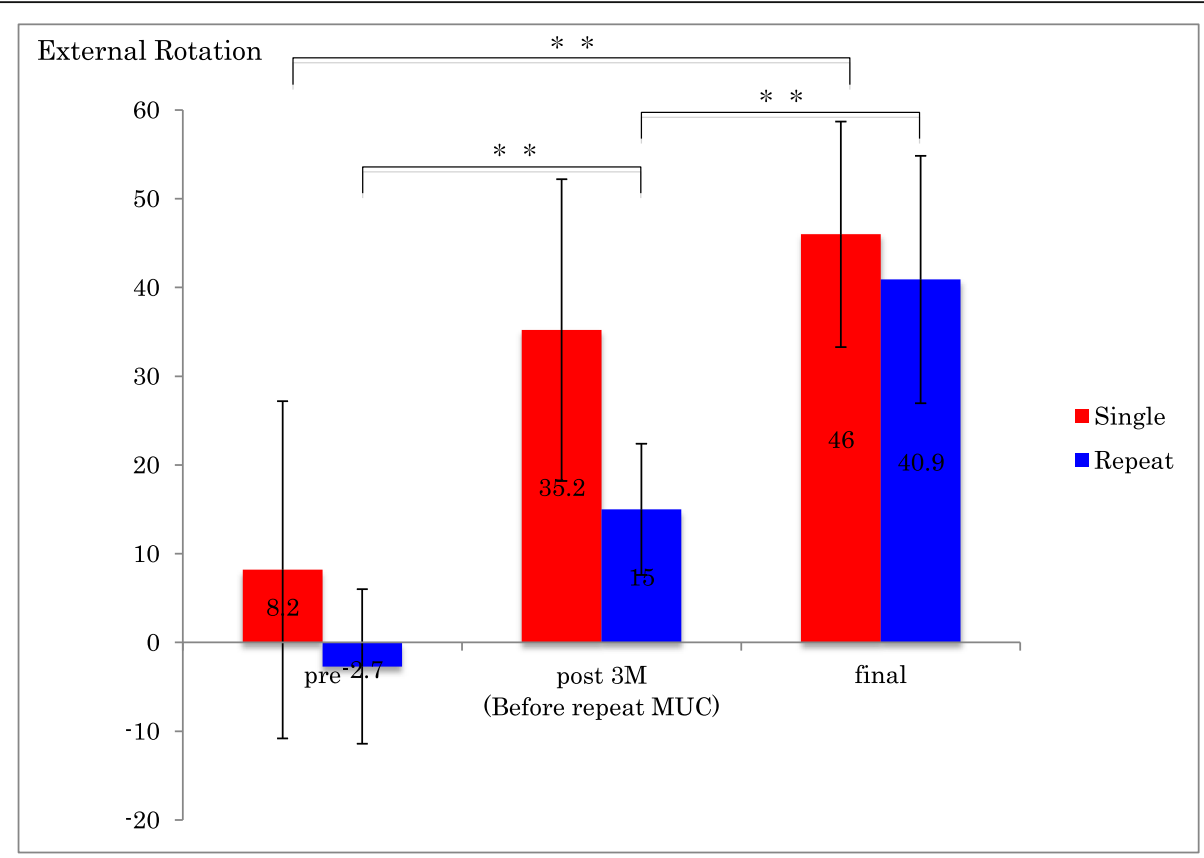

${ }^{* *} \mathrm{p}<0.01$

Fig 2 External rotation before and after single MUC and repeat MUC. ${ }^{* *} p<0.01$

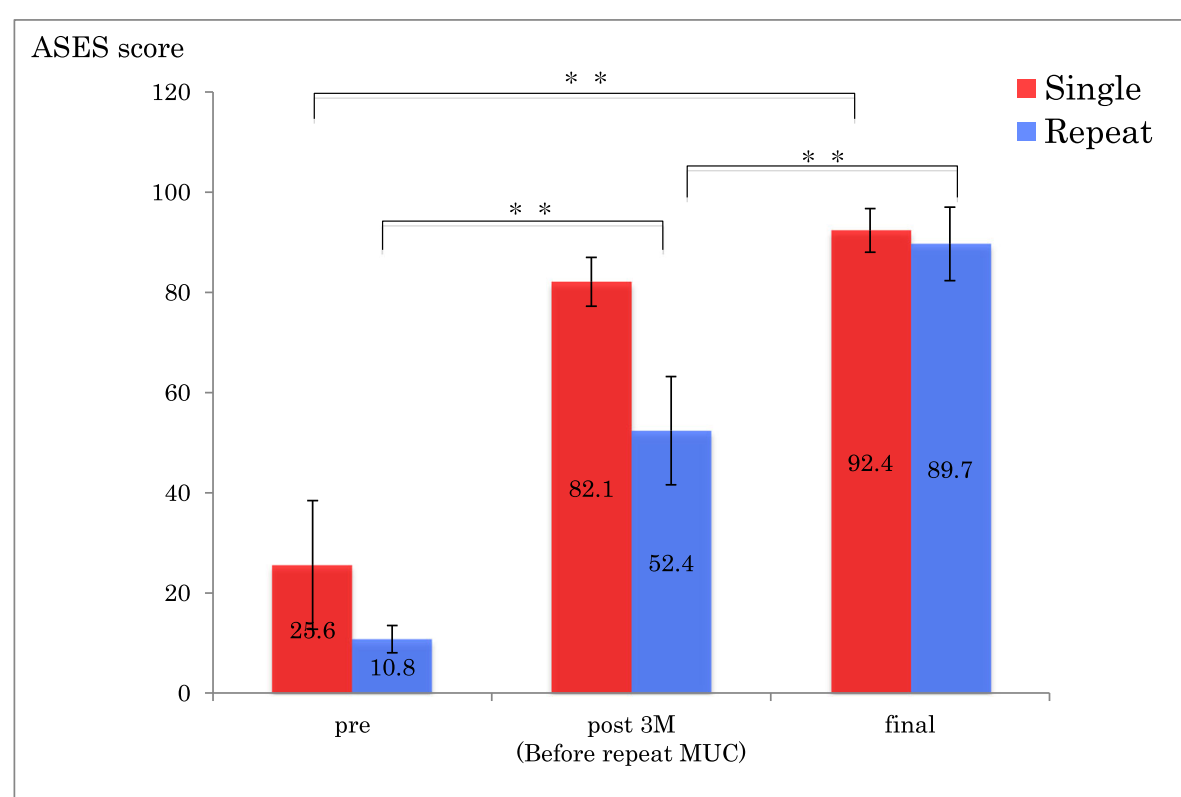

${ }^{* *} \mathrm{p}<0.01$

Fig 3 ASES score before and after single MUC and repeat MUC. ${ }^{* *} p<0.01$ 


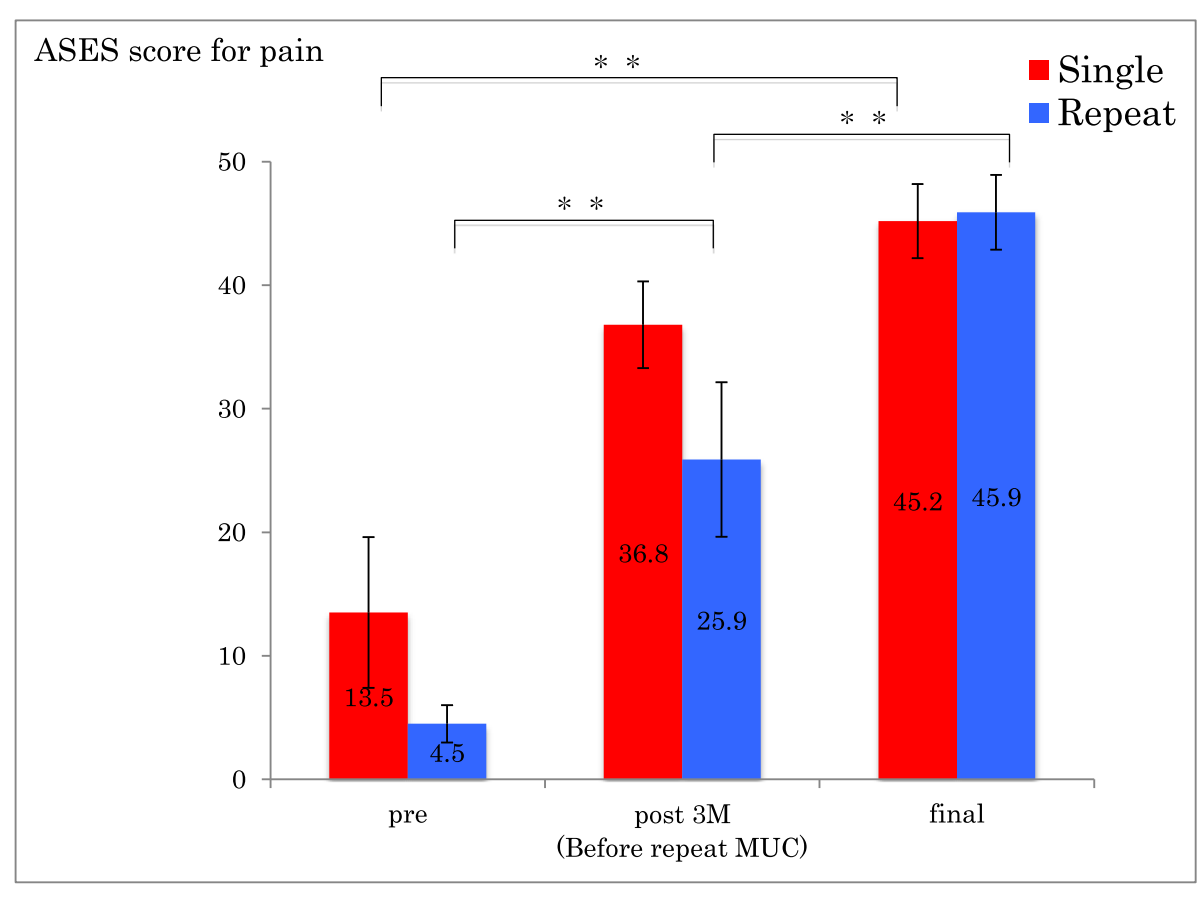

${ }^{* *} \mathrm{p}<0.01$

Fig 4 ASES score for pain before and after single MUC and repeat MUC. ${ }^{* *} p<0.01$

\section{Comparison of patient characteristics}

The requirement for a repeat MUC did not appear to be related to age, sex or duration of symptoms between the single and repeat groups. However, compared with the single group, the repeat group had revealed a significant severely limited ROM $(p<0.01)$ and a decrease in ASES score before the procedure $(p<0.01)$ (Table 1$)$.

\section{Complications}

There were no complications, such as fracture or dislocation, and no symptoms of acute rotator cuff tear, neurological or other iatrogenic injuries.

Table 1 Patient characteristics

\begin{tabular}{llll}
\hline & Single & Repeat & $\boldsymbol{P}$ \\
\hline Mean age & $53 \pm 7$ & $53 \pm 7$ & NS \\
Sex (female/male) & $22 / 8$ & $9 / 1$ & NS \\
Duration of symptoms (months) & $5.4 \pm 2$ & $5.5 \pm 2$ & NS \\
FF before MUC & $103.7 \pm 17$ & $80.4 \pm 22$ & 0.007 \\
ER before MUC & $8.2 \pm 16$ & $-2.7 \pm 7$ & 0.005 \\
ASES score before MUC & $25.6 \pm 12$ & $10.8 \pm 2$ & 0.0001 \\
\hline
\end{tabular}

$F F$ forward flexion, $E R$ external rotation, $M U C$ manipulation under ultrasoundguided cervical nerve root block, ASES American Shoulder and Elbow Surgeons, NS not significant

\section{Discussion}

This study confirmed that a repeat MUC with corticosteroid and local anaesthetic injection for recurrence of idiopathic frozen shoulder after MUC results in sustained improvement in ROM and ASES score. Patients who received a repeat MUC showed significantly severely limited ROM and a decreased ASES score before the procedure. There were no complications in our study.

The pathophysiology of frozen shoulder has been thought to be a combination of synovial inflammation, capsular fibrosis and chondrogenesis [19]. Cytokines and growth factors related to fibrosis and inflammation increase in joint capsule from frozen shoulder [19]. Yinghua et al. [20] suggested that neoinnervation and neoangiogenesis in the shoulder capsule are important events in the pathogenesis of frozen shoulder and help explain often-severe pain that patients with frozen shoulder experience.

Two recent studies have reported success with MUC for idiopathic frozen shoulder [17, 18]. Both studies performed MUC with corticosteroid and local anaesthetic injection. Sasanuma et al. [18] performed MUC in 30 shoulders and revealed MR imaging evidence of capsular tear in 29 shoulders (96\%). Loew et al. [21] performed arthroscopy in 30 shoulders after closed MUA and 
reported an $80 \%$ incidence of capsular tear in 24 shoulders. These results suggest that MUC procedure may have contributed to joint mobility and pain relief by rupturing the joint capsule which is one of main pathologies of frozen shoulder.

Furthermore, there is suggestion that intra-articular steroid injection may be beneficial in the short term for frozen shoulder but the effect may be limited and not well maintained [19]. Steroid injection is administered to reduce the synovial inflammation to inhibit capsular fibrosis [22]. Therefore, the addition of corticosteroid and local anaesthetic injection may have contributed to reducing inflammation and pain relief.

In a series of reports by Saito et al. [17], the adjusted ASES score at 1-year follow-up was, on average, $93 \pm 9$ points, which was similar to our results. On the other hand, in our study, 11 of 42 shoulders (26\%) developed recurrent moderate pain and limited range of motion after the procedure.

Generally, if patients continue to report significant pain, loss of range of motion and functional impairment after MUA, more aggressive interventions such as open or arthroscopic capsular release or manipulation under general anaesthesia may be indicated and most studies show some benefit $[16,23,24]$. However, such techniques are more invasive and require general anaesthesia and hospitalisation.

To the best of our knowledge, there have been no studies involving the treatment of patients who fail to improve after MUC, nor have there been any published results regarding repeated MUC.

In our study, a total of 11 shoulders (26\%) required a repeat MUC if they had recurrence at 3 months after the procedure. We found that ROM (forward flexion, external rotation), ASES scores and ASES score for pain showed significant improvement after the initial and the repeat MUC. In a prospectively collected study, Woods and Loganathan [23] performed MUA in 730 patients with frozen shoulder. A total of 141 patients (17.8\%) required a further MUA. This study found that there was an initial improvement in the Oxford Shoulder Score (OSS), which subsequently deteriorated by the time of the further MUA, after which the scores significantly improved, these finding are consistent with our results.

In addition, they reported that in those patients who required a further MUA, the pre-operative ROM was greater than it had been before the initial MUA [23]. In our study, in patients who required repeat MUC, the pre-operative ROM of forward flexion, external rotation, ASES score and ASES score for pain were greater than before the initial MUC. These results suggest that if patients continue to report significant pain and limited range of motion after MUC, repeat MUC leads to improvement not only in range of motion but also in shoulder pain.

In our study, patients in the repeat group displayed significantly more severely limited ROM and worse ASES scores before their initial MUC compared with those in the single group. This is in contrast to the study by Woods and Loganathan [23], who found that there was no significant difference in mean pre-operative ROM between the groups for initial MUA. The exact cause is unclear, though muscle contraction or other connective tissue responses might have been more severe in our repeat group. Furthermore, arthroscopic capsular release allows precise and controlled release of the capsule and ligaments [19]. Closed manipulation, on the other hand, is associated with concerns regarding inadequate release of the articular capsule. The possibility that the articular capsule and ligament were more severe in our repeat group cannot be ruled out.

We recognise there are several limitations in our study. First, this was a retrospective study design. Second, our patient population was smaller than other studies. Third, the follow-up period in this study was quite short term; a longer-term follow-up is necessary to evaluate the efficacy of a repeat MUC.

\section{Conclusion}

In conclusion, patients with idiopathic frozen shoulder treated with MUC had significant improvement in shoulder ROM, pain and shoulder function. If patients continue to have pain and limited ROM after MUC, a repeat MUC with corticosteroid and local anaesthetic injection can be a valuable option before proceeding to surgery. A repeat MUC is likely to be required when there is severely limited ROM and decreased ASES score before the procedure, and this should be explained to the patient and their informed consent obtained.

\section{Abbreviations \\ MUC: Manipulation under ultrasound-guided cervical nerve root block; ASES: American Shoulder Elbow Surgeon; ROM: Range of motion; MUA: Manipulation under anaesthesia; FF: Forward flexion; ER: External rotation; OSS: Oxford Shoulder Score}

\section{Acknowledgements}

We thank Yutaka Mifune MD for his helpful advice with this study and Geoff Rupp for language revision.

\section{Authors' contributions}

TO participated in establishing the research design, data collection, data processing and data analysis as well as completing the manuscript. MY and $\mathrm{KH}$ participated in the research design, data collection, data proceeding, data interpretation and editing the manuscript. KP participated in establishing the research design and MUC procedure. All authors have read and approved the final manuscript.

Funding

There is no funding source.

Availability of data and materials Not applicable. 


\section{Ethics approval and consent to participate}

The study was approved by the institutional review boards of the authors' affiliated institutions, and informed consent was obtained from all subjects,

\section{Consent for publication}

Not applicable.

\section{Competing interests}

The authors declare that they have no competing interests.

\section{Author details}

'Yagi Orthopaedic Clinic, 47-1, Zike-machi, Kakogawa-cho, Kakogawa, Hyogo 675-0066, Japan. ${ }^{2}$ Paku Pain Clinic, 6-1-20, Gokodori, Chuo-ku, Kobe, Hyogo 651-0087, Japan.

Received: 7 August 2020 Accepted: 24 November 2020

Published online: 07 December 2020

\section{References}

1. Zuckerman JD, Rokito A. Frozen shoulder: a consensus definition. J Shoulder Elb Surg. 2011:20(2):322-5.

2. Diercks RL, Stevens M. Gentle thawing of the frozen shoulder: a prospective study of supervised neglect versus intensive physical therapy in seventyseven patients with frozen shoulder syndrome followed up for two years. J Shoulder Elb Surg. 2004:13(5):499-502.

3. Vastamäki H, Kettunen JPT, Vastamäki M. The natural history of idiopathic frozen shoulder a 2- to 27-year follow up study. Clin Orthop Relat Res. 2012; 470(4):1133-43.

4. Shafer B, Tibone JE, Kerlan RK. Frozen shoulder. A long-term follow-up. J Bone Joint Surg Am. 1992;74(5):738-46.

5. Farrell CM, Sperling JW, Cofield RH. Manipulation for frozen shoulder: longterm results. J Shoulder Elb Surg. 2005;14(5):480-4.

6. Jenkins EF, Thomas WJ, Corcoran JP, Kirubanandan R, Beynon CR, Sayers $A E$, et al. The outcome of manipulation under general anesthesia for the management of frozen shoulder in patients with diabetes mellitus. J Shoulder Elbow Sur. 2012;21(11):1492-8.

7. Vastamäki H, Vastamäki M. Motion and pain relief remain 23 years after manipulation under anesthesia for frozen shoulder. Clin Orthop Relat Res. 2013;471(4):1245-50

8. Reichmister JP, Friedman SL. Long-term functional results after manipulation of the frozen shoulder. Md Med J. 1999:48(1):7-11.

9. Hamdan TA, Al-Essa KA. Manipulation under anesthesia for the treatment of frozen shoulder. Int Orthop. 2003;27(2):107-9.

10. Thomas WJ, Jenkins EF, Owen JM, Owen JM, Sangster MJ, Kirubanandan R et al. Treatment of frozen shoulder by manipulation under anesthetic and injection: does the timing of treatment affect the outcome? J Bone Joint Surge Br. 2011:93-B(10):1377-81.

11. Holloway GB, Schenk T, Williams GR, Ramsey ML, lannotti JP. Arthroscopic capsular release for the treatment of refractory postoperative or postfracture shoulder stiffness. J Bone Joint Surg Am. 2001;83-A(11):1682-7.

12. Barnes $C P$, Lam PH, Murrell GA. Short-term outcomes after arthroscopic capsular release for adhesive capsulitis. J Shoulder Elbow Sur. 2016;25(9): e256-64.

13. Ranalletta M, Rossi LA, Zaidenberg EE, Campos C, Ignacio T, Maignon GD, et al. Midterm outcomes after arthroscopic anteroinferior capsular release for the treatment of idiopathic adhesive capsulitis. Arthroscopy. 2017;33(3): 503-8.

14. Le Lievre HM, Murrell GA. Long-term outcomes after arthroscopic capsular release for idiopathic adhesive capsulitis. J Bone Joint Surg Am. 2012;94(13): 1208-16.

15. Eid A. Miniopen coracohumeral ligament release and manipulation for idiopathic frozen shoulder. Int J Shoulder Surg. 2012;6(3):90-6.

16. Omari A, Bunker TD. Open surgical release for frozen shoulder: surgical findings and results of release. J Shoulder Elb Surg. 2001;10(4):353-7.

17. Saito T, Sasanuma H, lijima Y, Kanaya Y, Saito T, Watanabe H, et al. Shortterm clinical results of frozen shoulder treated with shoulder manipulation under ultrasound-guided cervical nerve root block at outpatient setting: a case series. J Othop Sci. 2017;22(2):275-80.

18. Sasanuma $H$, Sugimoto $H$, Kanaya $Y$, lijima $Y$, Saito T, Saito T, et al. Magnetic resonance imaging and short-term clinical result of severe frozen shoulder treated with manipulation under ultrasound-guided cervical root block. J Shoulder Elb Surg. 2016;25(1):e13-20.

19. Itoi E, Arce G, Bain Gl, Diercks RL, Guttmann D, Imhoff AB, et al. Shoulder stiffness: current concepts and concerns. Arthroscopy. 2016;32(7):1402-14.

20. Wu Y, Bonar F, Murrell GAC. Enhanced expression of neuronal proteins in idiopathic frozen shoulder. J Shoulder Elb Surg. 2012;21:1391-7.

21. Loew M, Heichel TO, Lehner B. Intraarticular lesions in primary frozen shoulder after manipulation under general anesthesia. J Shoulder Elb Surg. 2005:14:16-21.

22. Hannafin JA, Chiaia TA. Adhesive capsulitis. A treatment approach. Clin Orthop Relat Res. 2000:372:95-109.

23. Woods DA, Loganathan K. Recurrence of frozen shoulder after manipulation under anesthetic (MUA): the result of repeating MUA. Bone Joint J. 2017;99B(6):812-7.

24. Warner JJ, Allen A, Marks PH, Wong P. Arthroscopic release for chronic, refractory adhesive capsulitis of the shoulder. J Bone Joint Surg Am. 1996; 78(12):1808-16.

\section{Publisher's Note}

Springer Nature remains neutral with regard to jurisdictional claims in published maps and institutional affiliations.
Ready to submit your research? Choose BMC and benefit from:

- fast, convenient online submission

- thorough peer review by experienced researchers in your field

- rapid publication on acceptance

- support for research data, including large and complex data types

- gold Open Access which fosters wider collaboration and increased citations

- maximum visibility for your research: over $100 \mathrm{M}$ website views per year

At $\mathrm{BMC}$, research is always in progress.

Learn more biomedcentral.com/submissions 